Meta

Journal des tradlucteurs

Translators' Journal

\title{
Les banques de terminologie bilingues et multilingues : état de la question
}

\section{Carlos Del Burgo}

Volume 24, numéro 2, juin 1979

URI : https://id.erudit.org/iderudit/003982ar

DOI : https://doi.org/10.7202/003982ar

Aller au sommaire du numéro

Éditeur(s)

Les Presses de l'Université de Montréal

ISSN

0026-0452 (imprimé)

1492-1421 (numérique)

Découvrir la revue

Citer cet article

Del Burgo, C. (1979). Les banques de terminologie bilingues et multilingues :

état de la question. Meta, 24(2), 253-263. https://doi.org/10.7202/003982ar d'utilisation que vous pouvez consulter en ligne.

https://apropos.erudit.org/fr/usagers/politique-dutilisation/ 


\section{DROBLIEMES ET SOLUTIONS}

\section{PETIT VOCABULAIRE CONTEXTUEL DES MATIÈRES PLASTIQUES}

La source est abrégée entre parenthèses dans chaque cas; les références bibliographiques complètes sont énumérées après la liste d'équivalents, en regard des abréviations correspondantes.

\section{ANGLE PRESS}

See also : undercut

A hydraulic moulding press equipped with horizontal and vertical rams, and specially designed for the production of complex moldings containing deep undercuts (Frados 1976 2)

- la presse d'angle est une presse à deux pistons orthogonaux conçue pour le moulage des pièces dont la forme exige un moule en plusieurs parties (cas des pièces présentant une contre-dépouille inévitable) (Gossot 1968 127)

\section{BARREL}

A cylindrical housing in which the screw rotates (Frados 1976 10)

-- le corps de boudineuse, formé d'un cylindre dans lequel tourne la vis sans fin (Gossot 1968 108)

-- un fourreau chauffé (Aubineau 1974 204)

\section{BLOW MOLDING}

A method of fabrication in which a parison (hollow tube) is forced into the shape of the mold cavity by internal air pressure (ASTM 1976 347)

- c'est en plaquant le tuyau à la sortie de l'extrudeuse contre les parois d'un moule à l'aide d'air comprimé que l'on fabrique des corps creux, des bouteilles, par exemple (Universalis 131972 155)

- soufflage par pression de fluide (air ou eau chaude) (Gossot 1968 173)

-l'extrusion-soufflage (EIST 81972 955) 
terme à terme avec chacune des autres langues, ce qui constitue un travail gigantesque dont on ne voit pas bien la nécessité.

On est donc en droit de se demander si cette conception de la structure du fichier pourra répondre aux exigences des banques de terminologie du $\mathrm{XxI}^{\odot}$ siècle.

L'une des hypothèses que l'on envisage à l'heure actuelle comme solution possible consisterait à classer non pas des termes, mais des notions. Dans cette hypothèse, les notions seraient définies comme des ensembles de relations entre notions et s'exprimeraient donc à l'aide d'un métalangage. Chaque notion ainsi définie par rapport à un sous-ensemble des autres notions du même domaine serait alors reliée à l'appellation qui sert à la désigner en langue naturelle selon le schéma de la page précédente.

Ce tableau schématise l'hypothèse d'une définition des notions fondée sur un réseau de relations inter-notionnelles. Par exemple la notion $\mathrm{N}_{1}$ se définirait comme l'ensemble des relations (chacune accompagnée de son ensemble de traits distinctifs) qui la mettent en rapport avec les autres notions du même domaine. Dans cette optique, un domaine se définit comme un ensemble structuré de notions, ce qui rejoint l'un des postulats fondamentaux de la terminologie.

Le tableau montre également comment les notions peuvent être représentées par des appellations en langues naturelles, avec les cas où d'une part la même notion est représentée en deux langues naturelles par deux appellations qui se correspondent parfaitement et d'autre part où il ne trouve pas d'appellations en langues naturelles différentes correspondant exactement à la même notion. Le premier cas est illustré par la séquence $\mathrm{N}_{1} \rightarrow \mathrm{A}(\mathrm{F})=\mathrm{A}(\mathrm{I}) \rightarrow \mathrm{N}_{1}$ et le second cas par la séquence $N_{1} \rightarrow A(F) \neq A(E) \rightarrow N_{2}$ ou encore par la séquence $N_{1} \rightarrow A(I) \neq A(E)=A(F) \leftarrow N_{2}$, dans lesquelles $N_{n}$ se lit « la notion $N_{n} \gg$ et $\mathrm{A}$ (L) (pour $\mathrm{L}=\mathrm{E}$ (anglais), $\mathrm{F}=$ français, $\mathrm{D}=$ allemand, etc.) se lit « appellation en anglais, français, allemand », etc.

Des appellations en langues naturelles différentes sont en correspondance bi-univoque si et seulement si elles correspondent à une seule et même notion, indépendamment de leur forme, qui peut être identique ou non.

Mais cette hypothèse s'appuie sur le postulat de l'universalité des notions, défendu par une certaine école de pensée en terminologie et qui vient en contradiction avec les observations générales de la linguistique. Le débat reste donc entièrement ouvert et ce dossier s'ajoute à de nombreux autres qui alimenteront la recherche en terminologie dans les prochaines décennies.

GuY RONDEAU 
précautions, d'adopter pour la banque de terminologie le découpage de la réalité propre à la langue naturelle-pivot;

b) ensuite, cette conception rend très difficile l'introduction d'une troisième, d'une quatrième, etc., langue, car ou bien, pour chaque nouvelle langue, on établira des rapports de terme à terme qu'avec la langue-pivot, ce qui serait fausser la réalité, ou bien on devra établir ces rapports de

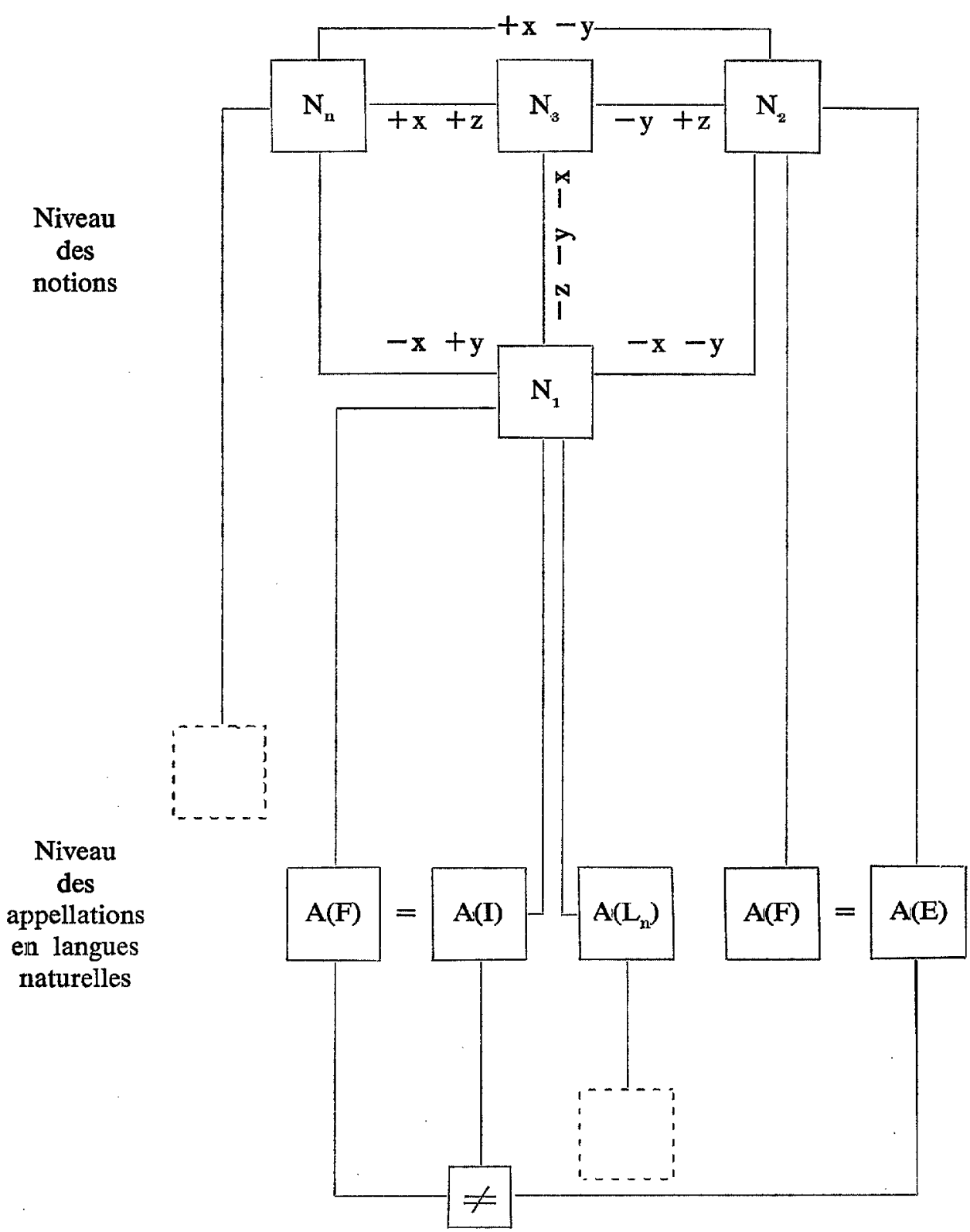


2. Problèmes de sémantique lexicale

3. Problèmes de morphologie en néologie

4. Problèmes de découpage du terme

5. Problème du contexte en terminologie

6. Les valeurs connotatives du terme

7. Les niveaux de langue en terminologie

8. Les emprunts de la langue commune aux langues techniques et de spécialités

9. Peut-on fonder un système de stockage/dépistage des données terminologiques sur des relations entre notions? entre termes ?

10. Peut-on concevoir un système universel de notions ?

Cette énumération de questions non encore résolues, pour fragmentaire qu'elle soit, suffit à démontrer l'ampleur des travaux qui attendent les terminologues dans les prochaines décennies et justifie pleinement, semble-t-il, l'intérêt que portent de plus en plus à la terminologie les universités québécoises.

Je voudrais, en terminant, aborder un problème des banques de terminologie de l'avenir, ne fut-ce que de façon superficielle, car le temps ne nous permettra pas d'aller très loin. J'aborderai la question du multilinguisme des fichiers, laissant de côté, évidemment, les problèmes de multilinguisme en terminologie, qui ne sont pas des problèmes de banque, mais de recherche terminologique.

C'est un problème auquel on n'a pas encore véritablement eu à faire face, car nos banques sont pour le moment, à toutes fins pratiques, expérimentales et à ce titre elles n'ont à traiter que quelques centaines de milliers de fiches. Mais le jour n'est pas loin où elles deviendront fonctionnelles et où, par conséquent, la masse des données à traiter augmentera considérablement. Or, la question du nombre de langues en usage dans une banque de terminologie a une influence directe sur la masse des données à traiter, car pour un millier de notions, on aura dans une banque bilingue au moins deux mille fiches et le problème devient encore plus complexe si l'on a affaire à trois langues.

Dans la génération actuelle des banques, la question a été résolue de façon pratique en adoptant le principe de la langue naturelle-pivot. C'est en vertu de ce principe que l'on parle de vedette et d'équivalent ou encore de langue de départ d'une fiche et de langue d'arrivée, s'inspirant en cela de la démarche du traducteur. Ainsi, dans les deux banques de terminologie en existence au Canada, c'est l'anglais qui joue le rôle de langue naturelle - terme français équivalent au terme anglais que l'on veut remplacer dans l'usage, il est normal qu'il en soit ainsi.

Mais cette conception comporte deux inconvénients majeurs :

a) d'abord, comme le découpage de la réalité ne se fait pas de la même manière d'une langue à l'autre, on risque, même en prenant toutes les 
n'en demeure pas moins importante, la question du coût, qui reste passablement élevé si on le calcule par questions, et plus élevé encore si on le calcule par réponses. Voir pour une discussion plus détaillée sur les modes d'interrogation, un article préparé en collaboration avec Jean-François Grégoire et Philippe Tessier et paru dans Meta, septembre 1977.

\section{Compatibilité}

Une autre caractéristique des banques de terminologie existantes, c'est l'orientation vers des besoins spécifiques, ce qui se traduit, on l'a vu, par des modes de construction et d'opération propres à chacune, d'où l'incompatibilité générale. C'est à cause de cette incompatibilité que, dans la première phase de l'établissement d'un réseau mondial de banques de terminologie, prévu pour 1979 ou 1980 , les échanges ne se feront pas directement de banque à banque, mais par le moyen de bandes magnétiques dont les données seront simplement lues et imprimées sans entrer dans le fichier de la banque receveuse. Un groupe de travail de l'ISO (Organisation internationale de normalisation) a été chargé de préparer une norme définissant les formats d'échanges sur bandes magnétiques de données terminologiques, tellement les différences sont importantes d'une banque à l'autre. C'est selon le schéma suivant que le réseau pourra fonctionner :

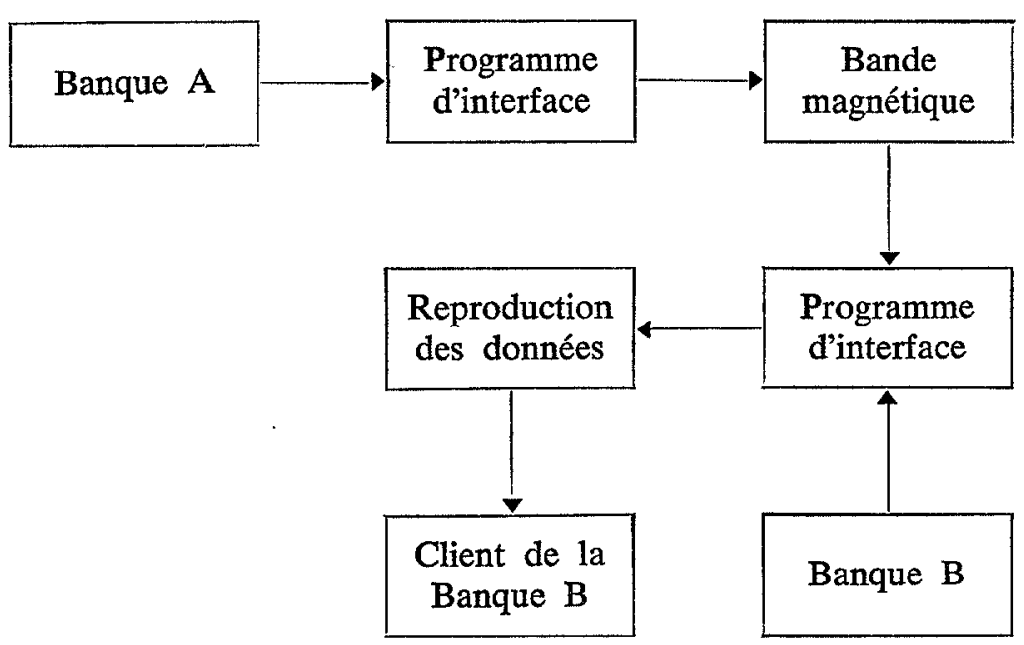

Comme on peut le constater, les banques de terminologie se sont développées de façon remarquable en très peu de temps.

Il reste cependant plusieurs problèmes à régler et place, par conséquent, pour la recherche. Permettez-moi d'énumérer sans plus quelques-uns de ces problèmes :

1. Le paradoxe entre le postulat terminologique de l'antériorité du classement des notions, postulat qui se vérifie dans un nombre relativement restreint de sciences exactes, et la réalité lexicologique des langues techniques et de spécialités 
naires dans le grand public. Même les dictionnaires purement descriptifs sont en effet perçus comme des arbitres du bon langage. À cause de la vaste diffusion dont fera l'objet leur contenu d'ici une dizaine d'années, à mesure que des masses de plus en plus importantes d'usagers y auront accès, à cause également du prestige qui s'attache à la machine, les banques de terminologie sont appelées à devenir un puissant moyen d'influence sur l'usage linguistique.

\section{Méthodes de création néologique}

Elles ont été mises au point à partir de l'observation des procédés naturels de création lexicale pour une langue donnée, de critères morphologiques comme la richesse de dérivation (ou la «dérivabilité ») et de facteurs sociolinguistiques comme l'《 intégrabilité » dans l'usage. Les recherches dans ce domaine n'en sont cependant encore qu'à leur début.

\section{Définition du mode d'entrée}

L'aspect terminologique de cette question a été traité au numéro un plus haut et se trouve concentré dans la fiche de saisie. L'aspect informatique est une simple question technique, de peu d'intérêt ici. Notons tout simplement qu'exception faite des fichiers transmis d'une banque à l'autre sur bande magnétique le procédé le plus couramment utilisé est le lecteur optique de caractères spéciaux en attendant que les développements technologiques permettent de lire n'importe quels caractères.

\section{Classement des données}

C'est probablement le point sur lequel les banques de terminologie accusent le plus de retard et c'est également un point crucial dans leur développement. À l'heure actuelle, chaque banque a son propre système de classement des données par domaines et aucun ne donne satisfaction.

On se trouve ici en face du problème, bien connu en linguistique et non encore résolu, de la sémantique lexicale. De la même manière que pour la traduction automatique la faiblesse de nos connaissances en sémantique a été un handicap, de la même manière, l'absence d'un modèle général et exhaustif de sémantique lexicale risque de nuire longtemps à l'efficacité des banques de terminologie.

\section{Modes d'interrogation}

Ce qui caractérise l'état actuel des banques de terminologie quant à cet aspect, c'est d'abord un taux important de bruit ou de silence involontaire. (On parle de bruit involontaire quand l'interrogateur reçoit une avalanche de données alors qu'il en attendait un petit nombre de soigneusement sélectionnées par le système ; on parle de silence involontaire quand le système ne fournit pas, parce que la question est «mal » posée, les données qu'il possède par ailleurs.) C'est ensuite la nécessité d'interroger la banque par l'intermédiaire d'un initié. En effet, les banques actuelles ne permettent pas l'interrogation directe par l'usager ordinaire. C'est, enfin, une considération fort peu linguistique, avouons-le, mais qui 
Au terme de ce bref tour d'horizon, on peut tenter d'établir le bilan des banques de terminologie existantes, puis de voir quels sont les problèmes qu'elles auront à résoudre dans les années à venir.

Notons d'abord qu'il ne faut pas confondre banque de terminologie et dictionnaire automatique. Dans ce dernier cas, il suffit de mettre en mémoire des données lexicographiques ou terminologiques dans un ordre alphabétique (qui devient numérique) pour les retrouver ensuite en suivant le même ordre.

Nous ferons le bilan des banques de terminologie sur huit points :

\section{Méthodes de dépistage et de collecte des données terminologiques}

Ces méthodes ont été mises au point et elles donnent satisfaction. Après de nombreuses rencontres sur le plan international, on s'est entendu sur le format général de l'outil principal de ce travail, la fiche de saisie des données en définissant les éléments qu'elle devait contenir. Ce modèle, défini en 1972, est adopté aujourd'hui par la plupart des banques existantes, avec des aménagements particuliers pour chacune et les banques en voie de création, comme celle du Danemark, emboîtent le pas.

Une exception à signaler : la banque de terminologie de la CEE à Luxembourg. Elle est conçue comme un outil à l'usage du traducteur. Ses méthodes de travail sont donc moins complexes que celles qui ont été développées au Canada, et qui correspondent à des besoins plus variés.

\section{Méthode de recherches terminologiques}

La terminologie a établi, dans ce domaine, une méthode originale qui se distingue nettement des méthodes lexicologiques, fondée uniquement sur le dépouillement de textes. La méthode propre à la terminologie se fonde sur le principe qu'il faut aller de la notion vers le terme. Elle consiste donc à délimiter d'abord un thème : discipline ou sous-discipline ou champ d'activités, puis à en faire le découpage notionnel en systématisation hiérarchique. Les termes prennent alors naturellement leur place respective dans le système hiérarchique et les "trous» sont comblés d'abord par le recours au dépouillement de textes puis, si nécessaire, au moyen de la création néologique. En terminologie bilingue, on établira un système hiérarchique pour chaque langue, ce qui permettra de comparer les termes d'une langue à l'autre dans l'optique de leur place respective dans les systèmes hiérarchiques.

\section{Normalisation terminologique}

C'est non seulement par son aspect sociolinguistique mais également par son aspect normatif que la terminologie se rapproche de la linguistique appliquée. La normalisation terminologique répond au besoin de la clarté de la communication dans les langues techniques et de spécialités. Elle consiste à supprimer la synonymie, à rejeter les doublets, selon le postulat qu'à une notion correspond une appellation et une seule pour un niveau de langue donné. L'action normalisatrice des banques de terminologie peut se comparer à celle des diction- 
La fonction « Entrée » comporte des subdivisions que l'on peut représenter de la façon suivante :

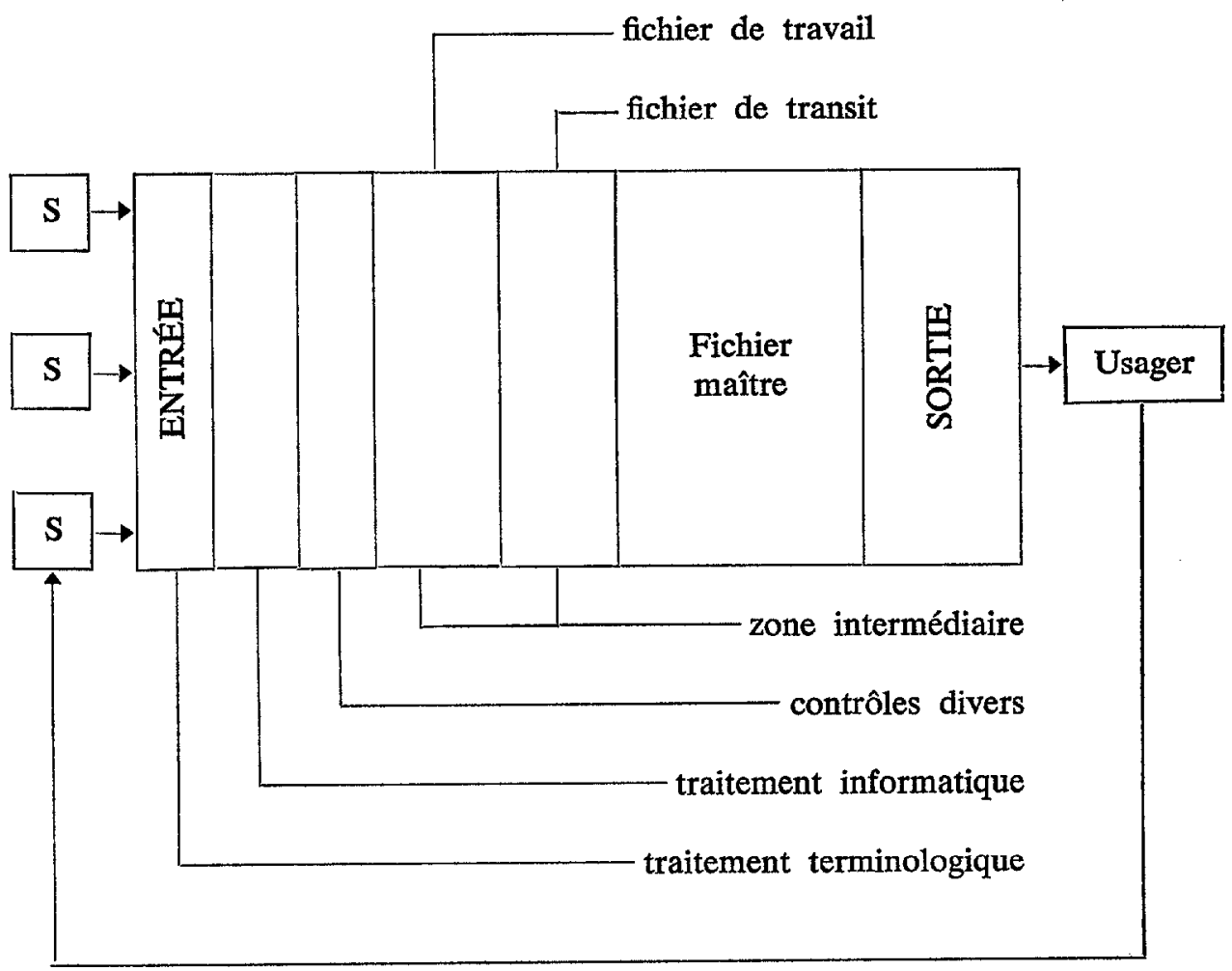

La fonction «Sortie » comporte des subdivisions que l'on peut représenter comme suit :

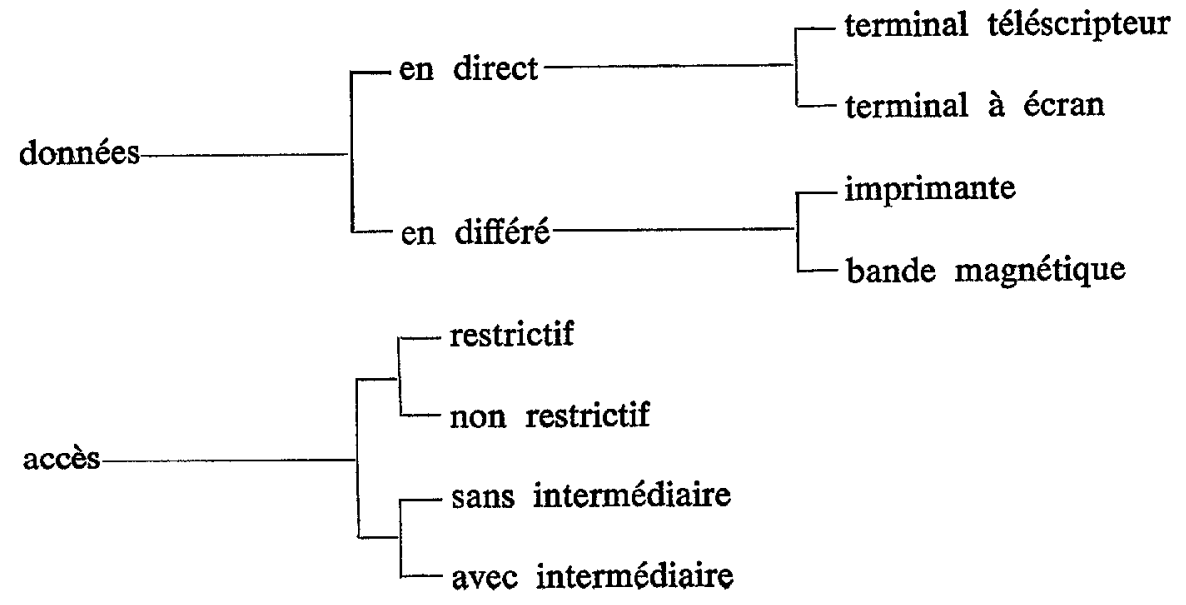


Voir à ce sujet : G. Rondeau, Bibliographie de la terminologie (à paraître).

La terminologie se distingue essentiellement de la lexicologie en ce sens qu'elle part d'un classement des notions pour aboutir aux termes alors que cette dernière fait le recensement des mots et expressions pour les noter avec leurs sens divers.

Sur le plan des méthodes de travail, la terminologie emprunte à l'occasion à la lexicologie et à la lexicographie, mais la terminologie se caractérise par une grande rigueur méthodologique qui va même jusqu'à la normalisation des procédés de lexicographie terminologique. Pour citer un exemple frappant des points de vue parfois diamétralement opposés des méthodes de la lexicographie traditionnelle et de celles de la lexicographie terminologique, considérons le fait que le seul mode de présentation du vocabulaire en usage en lexicographie traditionnelle est le classement par ordre alphabétique, alors que la lexicographie terminologique condamne ce système et préconise un mode de classement systématique, ou tout au moins mixte.

La terminologie est par définition normalisatrice et tend ainsi à faire disparaître la synonymie, alors que la lexicologie est descriptive.

Voilà donc très brièvement pour ce qui est de la terminologie. Voyons d'un peu plus près une troisième notion, celle des banques de terminologie.

Nous avons noté précédemment qu'il s'en crée de nouvelles chaque année. On trouvera une liste de celles qui ont été recensées jusqu'en 1976 dans une publication d'Infoterm parue en septembre dernier (Infoterm Series No 3 Verlag Dokumentation, München).

Essentiellement, une banque de terminologie c'est un ensemble composé de trois éléments correspondant chacun à une fonction et dont l'objectif est de faire la collecte, le stockage et la diffusion des données terminologiques le plus rapidement possible, avec le moins de bruit possible et au coût le moins élevé.

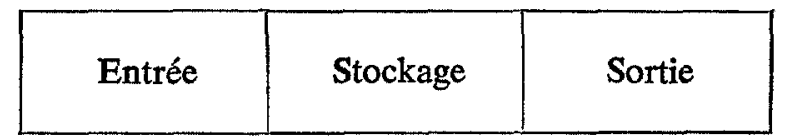

Le schéma qui précède illustre les trois composantes d'une banque de terminologie.

Évidemment, chaque banque doit répondre à des besoins spécifiques, ce qui détermine des choix particuliers quant à la sélection des données, quant aux modes d'entrée et de sortie, quant à l'aménagement des fichiers, quant aux types de relations établies entre les données, etc. Par exemple, toutes les banques de terminologie existantes sont au moins bilingues et celle de la CEE à Luxembourg comprend six langues et devra probablement bientôt en compter neuf. Ce n'est donc pas seulement des relations entre notions à l'intérieur d'une même langue qu'il faut établir, mais des relations entre termes d'une langue à l'autre. 
Ce qui rend parfois difficile pour le non-initié l'identification des termes, c'est l'emploi en langue technique de mots ou d'expressions empruntés à la langue commune. En d'autres termes, on joint à une notion $N$ une appellation existante, mais que l'on dépouille de ses valeurs sémantiques et stylistiques pour en faire un terme. Il arrive également qu'un terme passe de la langue technique à la langue commune ; on aura alors une appellation dont l'existence sera double, à la fois comme terme et comme mot de la langue commune. C'est le cas de radio, radar, transistor, etc.

S'il est relativement facile de circonscrire la notion de terme, il n'en va pas de même pour celle de terminologie. Bien qu'il se trouve à l'heure actuelle une documentation assez abondante sur la terminologie, bien que la terminologie ait fait l'objet depuis dix ans d'une quinzaine de colloques internationaux et nationaux en Europe et en Amérique, bien que l'Unesco ait mis sur pied un organisme, Infoterm, chargé de diffuser sur le plan international les terminologies, bien qu'un nombre de plus en plus important de terminologues fassent de la terminologie à temps complet, bien que la terminologie s'enseigne dans des universités et collèges d'Europe et d'Amérique, bien qu'il existe des banques de terminologie, malgré tout cela - et aussi paradoxal que cela puisse paraître - il n'existe pas de définition universellement acceptée de la terminologie.

En 1975, avait lieu au Lac Delage un colloque réunissant pendant cinq jours une quarantaine d'experts internationaux sur le thème : "Essai de définition de la terminologie». Il en est sorti, comme vous le savez, non pas une, mais deux définitions de la terminologie. On trouvera dans un article publié dans le Bulletin de l'ACLA, un recensement des définitions de la terminologie.

Qu'est-ce que cela signifie ? Tout simplement qu'on est en face d'une discipline nouvelle, en voie de formation et qui, comme toutes les disciplines, n'a pas besoin de définition pour exister. Voyons d'un peu plus près de quoi il s'agit.

Il faut noter tout d'abord qu'il n'y a pas un terme terminologie, mais plusieurs, et au moins trois :

1. L'ensemble des termes d'un domaine, comme par exemple la biochimie ou d'une discipline, comme par exemple la linguistique. On dira alors : la terminologie de la biochimie, de la linguistique, etc.

2. Les méthodes de collecte et de classement des termes, de création néologique, de normalisation des termes, de diffusion des termes : c'est ce à quoi s'emploient les terminologues et les terminographes.

3. Une science dont l'objet est d'ordre linguistique, mais qui est essentiellement pluridisciplinaire et participe à la fois de la linguistique, de la logique, de l'ontologie, de la classologie et de l'informatique. Les bases de cette science ont été jetées par le Prof. E. Wüster dans les années trente. Elles ont été reprises et développées principalement à l'Académie des sciences de Moscou et en Tchécoslovaquie dans les années soixante. On trouve sur le sujet une documentation abondante rédigée en russe, en allemand et en tchèque et, plus récemment, en anglais et en français. 
part la puissance des ordinateurs de la génération actuelle. Ce dernier aspect n'est pas négligeable, si l'on songe au volume énorme de données que les banques de terminologie devront bientôt manipuler, non seulement pour les mettre en mémoire, ce qui ne pose pas de problèmes, mais aussi et surtout pour les retrouver et les regrouper rapidement et à un coût raisonnable. (Pour citer un exemple, la Banque de terminologie du Canada contient à l'heure actuelle un million et demi d'entrées et ce n'est que le début du stockage.)

Mais avant d'aller plus loin, voyons d'un peu plus près les termes dont nous aurons à faire usage. Il semble utile, sinon de définir, du moins d'expliciter les concepts suivants : terme, terminologie, banque de terminologie.

Le terme est l'objet propre de la terminologie. La définition la plus courante qu'on en trouve est la suivante : ensemble des mots ou expressions propres à une science, une technique, une activité humaine. Plus précisément, un terme $\mathbf{T}$ est le produit du rapport entre une notion $N$ et une appellation $A\left(T=\frac{N}{A}\right)$, la notion étant délimitée par rapport à l'ensemble des notions constituant un domaine. Il faut noter également que la relation entre $\mathrm{N}$ et $\mathrm{A}$ est bi-univoque et permanente pour la durée de l'existence du terme. On constate que le terme est une unité linguistique possédant ses caractéristiques propres qui en font un sous-ensemble des lexèmes d'une langue. Les termes, répétons-le, se distinguent des mots ou expressions de la langue commune principalement par les traits suivants :

- découpage des notions par domaines

- bi-univocité et permanence de la relation $\mathrm{N} / \mathrm{A}$

- bi-univocité et réflexivité de la relation $T=\frac{N}{A}$

de sorte qu'à toute variation de $\mathrm{N}$ correspond une variation de $\mathrm{T}$

$$
\frac{\mathrm{N}+\mathrm{i}}{\mathrm{A}}=\mathbf{T}+\mathrm{i}
$$

Cette considération est fondamentale en terminologie. Si l'on a, en effet, $\frac{N}{A}=T$, pour peu qu'il y ait modification de $N$, même avec la même appellation, on aura un nouveau terme, homographe du premier. La terminologie ne connaît pas, théoriquement, de nuances de sens.

Ce postulat démontre par ailleurs le besoin de pousser plus avant les recherches théoriques en terminologie, dans le but de l'adapter à une réalité lexicale où tout n'est pas aussi nettement tranché que les fondateurs de la terminologie l'auraient souhaité.

Par ailleurs, de la même manière que pour la langue commune, $\mathrm{N}$ ou $\mathrm{A}$ peuvent être simples ou complexes. Exemples : monocotylédone, chlorure de sodium, morphème, labio-dentale, etc. 